\title{
Deep Vein Thrombosis and Pulmonary Embolism as a Complication of Traditional Chinese Acupuncture and Cupping
}

ThucNhi T. Dang Bsc, Albert A.C. Yeung MSc, MD

About the Authors
ThucNhi T. Dang is a 2nd year medical student at the University of Alberta Hospital,
Edmonton. Albert Yeung is a staff internist at the Royal Alexandra Hospital in
Edmonton, Alberta, and adjunct professor of pharmacology and medicine, University of
Alberta. Correspondence may be directed to albert.yeung@ualberta.ca.

\section{Summary}

We provide the first reported case of deep vein thrombosis with pulmonary embolism following acupuncture and cupping. This is a reminder that although serious adverse events associated with acupuncture and cupping are reportedly rare when performed by qualified practitioners, life-threatening complications can still arise.

\section{Résumé}

Nous présentons ici le premier cas signalé de thrombose veineuse profonde accompagnée d'une embolie pulmonaire par suite d'un traitement d'acupuncture et de l'application de ventouses. Ceci vient nous rappeler que même si les effets indésirables graves découlant de traitements d'acupuncture et de l'application de ventouses seraient plutôt rares lorsque réalisés par des praticiens qualifiés, il n'en demeure pas moins que des complications extrêmement graves peuvent survenir.

\section{Case Report}

A 72-year-old Caucasian man presented to the emergency department with a 1 day history of right-sided anterior pleuritic chest pain. He had accompanying mild dyspnea but no dizziness or syncope. He did not have hemoptysis, leg swelling, or leg pain at the time. His past medical history included a remote uncomplicated myocardial infarction, localized prostate cancer which was successfully treated by prostatectomy 10 years before, and longstanding non-progressive idiopathic sensory peripheral neuropathy characterized by persistent paraesthesia and discomfort in his feet for which he received treatment with acupuncture and cupping from an established practitioner of traditional Chinese medicine (TCM).

Before his presentation, he received a total of 10 electroenhanced acupuncture sessions spanning over a 2 month period. For his last 2 sessions, which were separated by a 1 week interval, he received acupuncture followed by cupping over his right popliteal fossa - specifically at the denoted acupuncture point "bladder forty" (BL-40) (Figure 1), the typical treatment site for peripheral neuropathy of the lower limbs. Negative pressure in the cup was achieved by means of a mechanical hand-pump connected to the cup. He received 


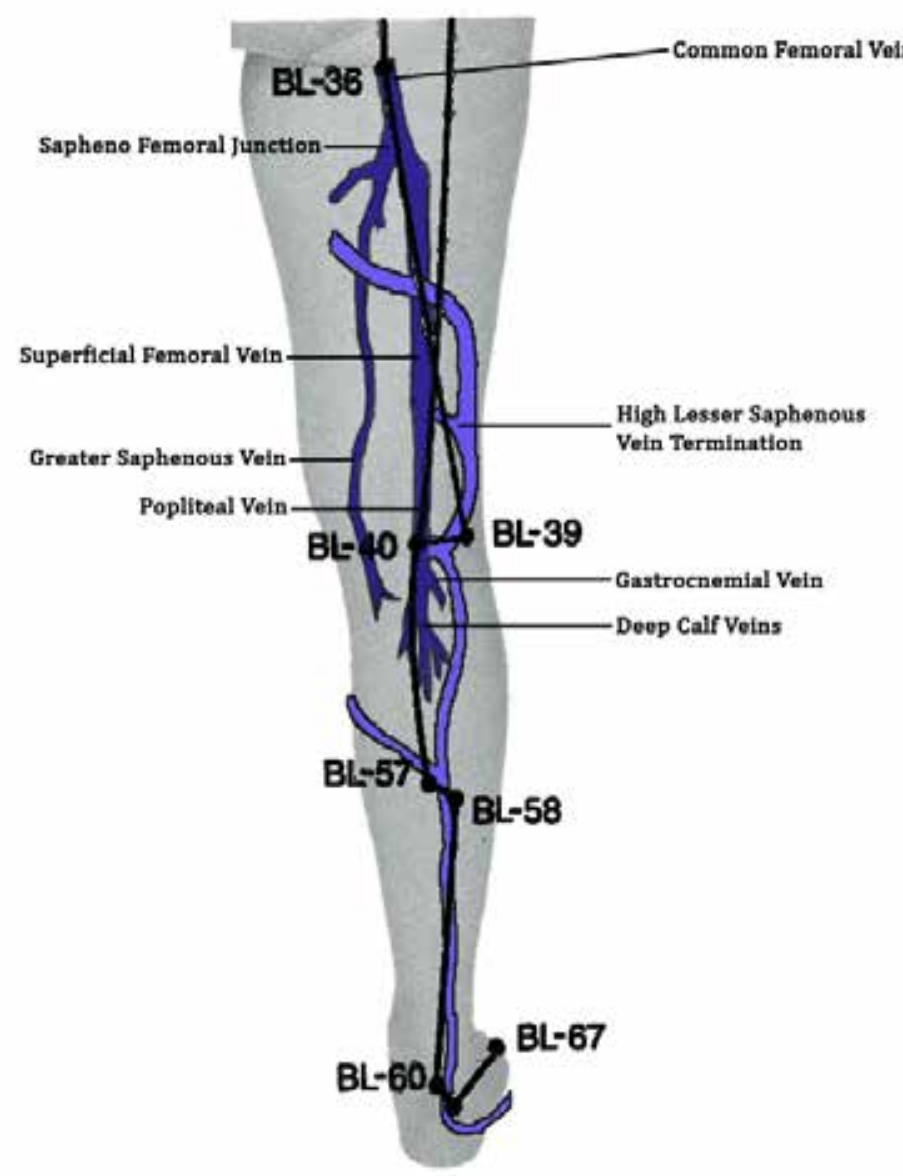

Figure 1. Acupressure point denoted BL-40 in relation to the venous anatomy of the right posterior leg and popliteal fossa.

his last treatment 6 days prior to the onset of chest pain, and he commented that the acupuncture and cupping session on that day went smoothly without local bleeding or undue discomfort.

The following day, he felt mild discomfort behind his right knee associated with a circular bruise over his popliteal fossa. Two days later, he complained of erythematous, tender swelling over the medial aspect of his right thigh, but he had no difficulty bending his knee or walking. Five days following the last treatment, his thigh pain and swelling had spontaneously resolved.

He denied prolonged stasis or recent trauma to his right leg. He had no personal history of thromboembolism. There was no family history of thromboembolism or thrombophilia. His only prescribed medication was atorvastatin $40 \mathrm{mg} /$ day. He was not taking any antiplatelet drug. He also regularly took a multivitamin tablet, fish oil, and coenzyme Q10 supplements.

On examination, he was in acute distress. Blood pressure $133 / 82 \mathrm{mmHg}$, heart rate 66 per minute, respiratory rate 23 per minute, oxygen saturation $89 \%$ on room air. There

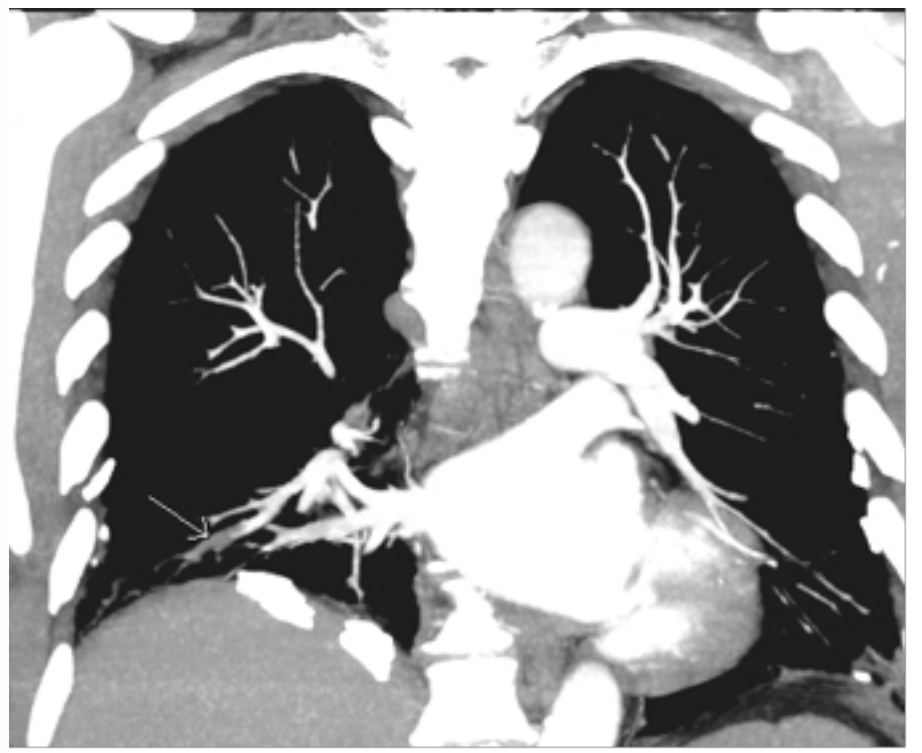

Figure 2. Enhanced computed tomography pulmonary angiogram showing a single right lower lobe pulmonary artery embolus (arrow)

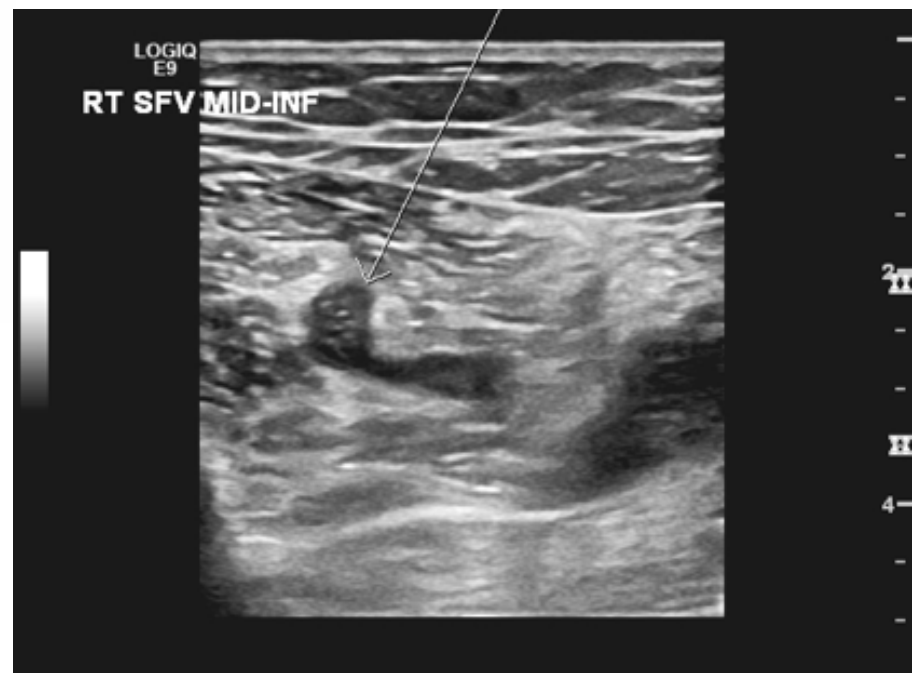

Figure 3. Doppler ultrasound showing residual thrombus of the intramuscular branch of the anterior thigh which feeds into the superficial femoral vein (arrow)

was no jugular venous distention. He had reduced air entry due to shallow inspiration. No pleural rub was heard. Leg examination showed no asymmetry, no swelling, nor bruising. Palpation along the popliteal vein did not elicit any tenderness.

Initial investigations revealed a normal complete blood count, creatinine, and coagulation panel. Serum troponin was not elevated, but the D-dimer assay was elevated at 1.4 $\mathrm{mg} / \mathrm{L}$ (fibrinogen equivalent units). Serum prostatic specific antigen was less than $0.1 \mathrm{ug} / \mathrm{L}$. Serum protein electrophoresis showed no monoclonal band. Thrombophilia testing for factor V Leiden and prothrombin G20210A mutations, activated protein $\mathrm{C}$ resistance, presence of antiphospholipid antibodies, 
and deficiencies in protein $\mathrm{C}$, protein $\mathrm{S}$, and antithrombin III were unremarkable. An enhanced computed tomography scan of the chest revealed a single segmental pulmonary embolus in the right anterolateral basal lobe (Figure 2).

Doppler ultrasound of his right lower limb showed a definite thrombus a few centimeters long within the lumen of a large intramuscular branch of the anterior thigh which drains into the superficial femoral vein (Figure 3). The common femoral and popliteal veins were patent.

He was treated with low molecular weight heparin subcutaneously followed by rivaroxaban orally. Eight weeks later, a repeat right leg ultrasound showed complete resolution of the deep vein thrombosis (DVT).

\section{Discussion}

TCM practitioners fundamentally believe that many diseases are caused by stagnant or blocked qi (pronounced chi) the vital energy or life force that normally flows along defined pathways in the body. Acupuncture and cupping are techniques believed to relieve blockages and correct imbalances in the flow of qi in order to restore health. ${ }^{1,2}$ Both acupuncture and cupping are becoming increasingly popular worldwide. ${ }^{3}$ The World Health Organization had identified acupuncture as an effective alternative therapy for 28 different disorders, although their recommendations were not evidence-based. ${ }^{4}$

We describe the first reported case of venous thromboembolism following acupuncture and cupping. A causal relationship is suggested by the temporality of events and the absence of any other identifiable risk factors for venous thromboembolism. The patient had not taken low dose ASA or alternative antiplatelet drug for over a year; however, he was on atorvastatin which has been reported to have some protective effect against DVT and pulmonary embolism. ${ }^{5,6}$ His heart rate had been relatively slow since his previous myocardial infarction; this could account for the absence of tachycardia on admission.

Virchow's triad of vascular thrombosis includes hypercoagulability, hemostasis, and endothelial injury. In this patient, the last factor was likely operative. Electroacupuncture needling may cause direct trauma to an underlying vein. In contrast, electroacupuncture in combination with cupping may additionally induce tissue edema or inflammation around veins. In our case, the absence of immediate bleeding from the acupuncture needling site or popliteal fossa swelling argues against direct venous puncture.

The overall risk of serious adverse events following acupuncture is reported to be very low. ${ }^{7-12}$ For instance, a review of 12 surveys of approximately 100,000 patients and over 1 million acupuncture treatments estimated the risk of serious adverse events to be $0.05 / 10,000$ treatments, or $0.55 / 10,000$ patients. ${ }^{7}$ The most common complications were pneumothorax, injury to the central nervous system, and infections, mostly hepatitis B. The commonest local infection was that of the external ear after auricular acupuncture. Complications were largely dependent on the treatment site, the depth of needle insertion, and the technical skill of the practitioner. A review of acupuncture experience in the United Kingdom, reported to the National Patient Safety Agency, found $95 \%$ of incidents to be inconsequential or of low clinical importance. ${ }^{11}$ The most serious adverse events included retained needles, dizziness, and fainting episodes. However, there has never been record of any instances of thromboembolism.

A careful 6 year survey of 62,482 acupuncture and moxibustion treatments performed at a national acupuncture clinic in Japan reported an adverse event rate of 14/10,000 treatment sessions. ${ }^{12}$ Failure to remove needles, ecchymosis, and burn injuries were among the most common complications. The authors expressed concern about the under-reporting of adverse events by acupuncture practitioners, especially in English language journals. Nevertheless, they concluded that severe or serious adverse events were rare in standard practice by trained practitioners.

Remarkably, acupuncture-induced vascular injuries have been singularly rare despite the widespread and increasing use of acupuncture worldwide., ${ }^{8,13,14}$ In 1991, the first case of acupuncture-induced DVT was reported..$^{15}$ Furthermore, Bergquist's systematic review of the literature on vascular injuries caused by acupuncture found only 2 cases with venous thrombosis - one calf DVT and a left crural vein thrombophlebitis. ${ }^{8,13}$ Traumatic bleeding and pseudoaneurysm formation were among the commonest vascular complications. ${ }^{13,14}$

Cupping, in contrast, is a TCM technique in which a vacuum is created over specific sites on the skin with a cup, sucking underlying tissue part way into the cup. The vacuum can be induced by heating enclosed air under the cup with a flame and allowing it to cool, or mechanically with a hand pump attached to the cup. The high tensile stress induced in the skin layer under the cup rim and stretched skin can cause capillary breakage with resulting erythema and ecchymosis in a characteristic circular pattern. The transmission of tension downwards becomes progressively reduced. ${ }^{16}$ Bruising caused by cupping may take days to weeks to dissipate.

Of the 7 major types of cupping used in China, our patient received "needle cupping." This consists of applying 
acupuncture needling first, followed by cupping for several minutes. Cupping is considered very safe, although the methodological quality of published reports attesting to its clinical efficacy and safety is weak. ${ }^{17}$ An unexplained D-Dimer elevation in the absence of thromboembolism was reported in a one report; ${ }^{18}$ another case report discussed the possible link between cupping to the cervical area and subsequent stroke from intracerebral hemorrhage in a non-hypertensive individual. ${ }^{19}$

\section{Conclusion}

Our case highlights the uncommon but potentially lifethreatening complication of venous thromboembolism following needle cupping. This is a reminder that although serious adverse events associated with acupuncture and cupping are reportedly rare, particularly when performed by qualified practitioners, life-threatening complications can still arise.

\section{Acknowledgments}

We would like to thank Dr. Steven K.H. Aung, MD for kindly helping us with his expert knowledge of acupuncture in the practice of Traditional Chinese Medicine, especially for Figure 1.

\section{References}

1. Chang, S., The meridian system and mechanism of acupuncture-a comparative review. Part 1: the meridian system. Taiwan J Obstet Gynecol, 2012. 51(4): p. 506-14.

2. Chang, S., The meridian system and mechanism of acupuncture--a comparative review. Part 2: mechanism of acupuncture analgesia. Taiwan J Obstet Gynecol, 2013. 52(1): p. 14-24. 